\title{
In silico design and synthesis of targeted rutin derivatives as xanthine oxidase inhibitors
}

\author{
Neelam Malik', Priyanka Dhiman ${ }^{1}$ and Anurag Khatkar ${ }^{2^{*}}$ (i)
}

\begin{abstract}
Background: Xanthine oxidase is an important enzyme of purine catabolism pathway and has been associated directly in pathogenesis of gout and indirectly in many pathological conditions like cancer, diabetes and metabolic syndrome. In this research rutin, a bioactive flavonoid was explored to determine the capability of itself and its derivatives to inhibit xanthine oxidase.

Objective: To develop new xanthine oxidase inhibitors from natural constituents along with antioxidant potential.

Method: In this report, we designed and synthesized rutin derivatives hybridized with hydrazines to form hydrazides and natural acids to form ester linkage with the help of molecular docking. The synthesized compounds were evaluated for their antioxidant and xanthine oxidase inhibitory potential.

Results: The enzyme kinetic studies performed on rutin derivatives showed a potential inhibitory effect on $\mathrm{XO}$ ability in competitive manner with $I_{50}$ value ranging from 04.708 to $19.377 \mu \mathrm{M}$ and $\mathbf{R U} \mathbf{Z a}_{\mathbf{3}}$ was revealed as most active derivative. Molecular simulation revealed that new rutin derivatives interacted with the amino acid residues PHE798, GLN1194, ARG912, GLN 767, ALA1078 and MET1038 positioned inside the binding site of XO. Results of antioxidant activity revealed that all the derivatives showed very good antioxidant potential.
\end{abstract}

Conclusion: Taking advantage of molecular docking, this hybridization of two natural constituent could lead to desirable xanthine oxidase inhibitors with improved activity.

Keywords: Rutin, Xanthine oxidase, Molecular docking, Antioxidant

\section{Introduction}

Xanthine oxidase (XO) having molecular weight of around $300 \mathrm{kDa}$ is oxidoreductase enzyme represented in the form of a homodimer. Both the monomers of $\mathrm{XO}$ are almost identical and each of them contains three domains namely (a) molybdopterin (Mo-pt) domain at the C-terminal having 4 redox centers where oxidation takes place (b) a flavin adenine dinucleotide (FAD) domain at the centre generally considered as binding site domain and (c) $2[\mathrm{Fe}-\mathrm{S}] /$ iron sulfur domain at the $\mathrm{N}$-terminal [1-3]. The catalytic oxidation of $\mathrm{XO}$ is two substrates reaction

\footnotetext{
*Correspondence: dranuragkhatkarmdurtk@gmail.com; anuragpharmacy@gmail.com

${ }^{2}$ Laboratory for Preservation Technology and Enzyme Inhibition Studies, Department of Pharmaceutical Sciences, M.D. University, Rohtak, Haryana, India

Full list of author information is available at the end of the article
}

on the xanthine and oxygen at the enzymatic centre. While xanthine undergoes oxidation reaction near to the Mo-pt center/substrate binding domain of XO, simultaneously substrate oxygen undergoes reduction at FAD center and electron transfer takes place leading to formation of superoxide anion $\left(\mathrm{O}^{2-}\right)$ or hydrogen peroxide $\left(\mathrm{H}_{2} \mathrm{O}_{2}\right)$ free radicals. [4-8]. This catalytic reaction results in formation uric acid as a final product and oxygen reactive species in form of free radicals. The excessive generation of uric acid leads to a condition like hyperuricemia which is a key factor in development of gout $[1,9]$, and uncontrolled amounts of reactive oxygen species causes many pathological conditions like cardiovascular disorders, inflammatory diseases and hypertensive disorders. Xanthine oxidase (XO; EC 1.17.3.2) has been considered as significantly potent drug target for the cure and management of pathological conditions prevailing due to high levels of uric acid in the blood stream. [10-17].

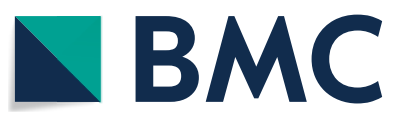

(c) The Author(s) 2019. This article is distributed under the terms of the Creative Commons Attribution 4.0 International License (http://creativecommons.org/licenses/by/4.0/), which permits unrestricted use, distribution, and reproduction in any medium, provided you give appropriate credit to the original author(s) and the source, provide a link to the Creative Commons license, and indicate if changes were made. The Creative Commons Public Domain Dedication waiver (http://creativecommons.org/ publicdomain/zero/1.0/) applies to the data made available in this article, unless otherwise stated. 
Considering the above fact, by inhibiting XO selectively could be better treatment plan for disorders caused by $\mathrm{XO}$ directly or indirectly including gout, inflammatory disease, oxidative damage and cancer [3, 18, 19]. Generally, $\mathrm{XO}$ inhibitors have been categorized into purine and non-purines inhibitors differentiated on the basis of their chemically derived skeleton structure. The first purine derived $\mathrm{XO}$ inhibitor discovered and approved by US FDA was Allopurinol as marketed drug for gout and hyperuricemia [20, 21]. Considering the life threatening side effects like Stevens-Johnsons syndrome caused by allopurinol use, scientists turned their interest into nonpurine $\mathrm{XO}$ inhibitors and an immense accomplishment has been received in this direction with development of new drug Febuxostat [22-25]. This non-purine candidate produced minor and non-life threatening adverse effects in comparison to Allopurinol [26-29]. Extending our previous successful effort to achieve new xanthine oxidase inhibitors from natural sources, in this report we investigated and developed some new rutin derived xanthine oxidase inhibitor [30].

Rutin is a well characterized bioactive plant flavonoid having great therapeutic importance for the treatment of many disease like conditions including cytotoxicity, antioxidant activity, antibacterial property and anti-inflammatory action [31-34]. Due to these pharmacological activities rutin is explored widely and great success have been achieved in order to get drug like candidates.<smiles>CC1OC(OCC2OC(Oc3c(-c4ccc(O)c(O)c4)oc4cc(O)cc(O)c4c3=O)C(O)C(O)C2O)C(O)C(O)C1O</smiles>

Rutin

Taking advantage of molecular docking techniques new compounds with potential drugability for the targeted enzyme might be achieved with a precise knowledge of mechanism of action. With the combined approach of molecular docking and synthetic chemistry, in this research we developed some new potential compounds against xanthine oxidase (Fig. 1).

\section{Experimental}

\section{Chemicals and instrumentation}

For this research, the analytical grade chemicals necessary for synthesis and antioxidant activity were purchased from Hi-media Laboratories. The in vitro evaluation of the human xanthine oxidase inhibitory activity was performed by measuring hydrogen peroxide $\left(\mathrm{H}_{2} \mathrm{O}_{2}\right)$ production from oxidation of xanthine oxidase by the substrate xanthine, utilizing the human xanthine oxidase assay kit (Sigma USA). The progress of reaction was observed through thin layer chromatography (TLC) on $0.25 \mathrm{~mm}$ precoated silica gel plates purchased from Merck, reaction spots were envisaged in iodine compartment and UV. Melting points were measured using a Sonar melting point apparatus and uncorrected. ${ }^{1} \mathrm{H}$ NMR and ${ }^{13} \mathrm{C}$ NMR spectra were documented in DMSO and deuterated $\mathrm{CDCl}_{3}$ respectively on Bruker Avance II 400 NMR spectrometer at the frequency of $400 \mathrm{MHz}$ using tetramethylsilane standard (downfield) moreover chemical shifts were expressed in ppm $(\delta)$ using the residual solvent line as internal standard. Infrared (IR) spectra were recorded on Perkin Elmer FTIR spectrophotometer by utilizing $\mathrm{KBr}$ pellets system.

\section{Molecular docking}

In silico docking studies was done with integrated Schrodinger software using Glide module for enzyme ligand docking [35].

\section{Protocol followed for docking procedures}

Preparation of protein The 3D crystal structure of human xanthine oxidase co-crystalised with salicylic acid was retrieved from Protein Data Bank (PDB ID. 2E1Q). The targeted protein structure was further refined in the Protein Preparation Wizard to obtain the optimized and chemically accurate protein configuration. For that, the co-crystalised enzyme (XO) was retrieved directly from Protein data bank in maestro panel followed by removal of water molecules, addition of $\mathrm{H}$ atoms, addition of missing side chains and finally minimization was done to obtain the optimized structure.

Preparation of ligand The 3D-structures of rutin derived compounds to be docked against $\mathrm{XO}$ were built in maestro building window. Ligand preparation was performed in Ligprep module.

Active site prediction To predict the binding site/active site Site Map application of glide was utilized. Out of top three active site, the one having larger radius was selected. Validation of binding site was done by redocking the salicylic acid and RMSD value was observed. RMSD value of less than 0.2 validated the docking procedure and active site was defined for docking of new rutin analogs.

Glide docking To carry out docking, Firstly the receptor grid generation tool was utilized to around the active/ 


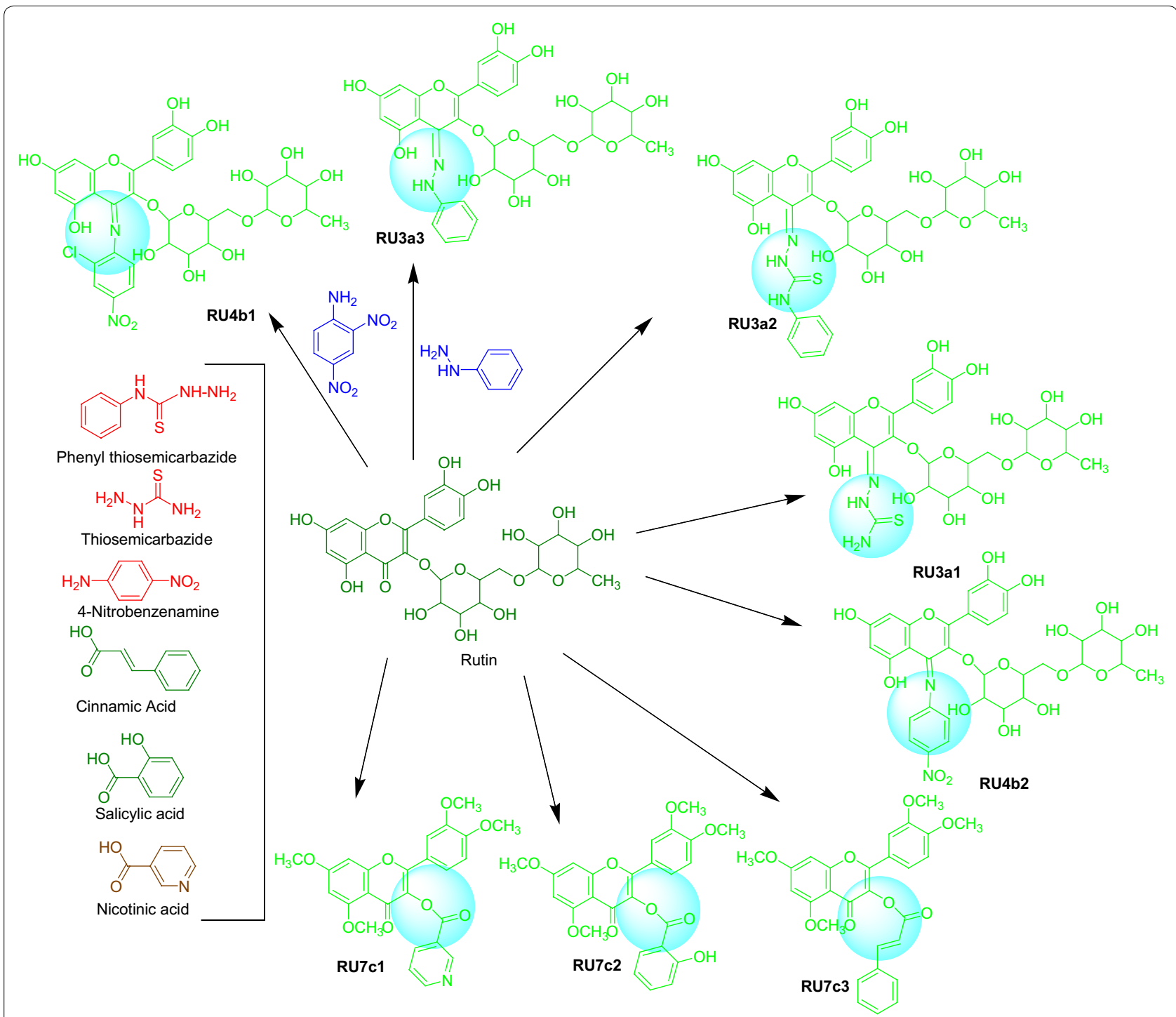

Fig. 1 Design strategy for the development of rutin derivatives

binding site of xanthine oxidase and glide docking with extra precision was used to visualize the interaction of protein and ligand. The top active ligand was selected for wet lab synthesis and evaluation of pharmacological activity.

\section{Synthetic procedures}

\section{Procedures for synthesis of rutin derivatives (Scheme 1)}

(A) General procedure for synthesis of hydrazine derivatives $R U 3 a_{(1-4)}$

$0.001 \mathrm{~mol}$ of rutin was taken in round bottom flask and dissolved in $50 \mathrm{ml}$ of ethanol. Different hydra- zines $(0.001 \mathrm{~mol})$ were added to the flask and reaction mixture was refluxed for $5-6 \mathrm{~h}$ at $40^{\circ} \mathrm{C}$. Completion of reaction was monitored by TLC. The product thus obtained was filtered and filtrate was concentrated to obtain the final product. The final product was recrystallised to obtain the pure compound.

(B) General procedure for synthesis of anilline derivatives RU4b $(1-2)$

$0.001 \mathrm{~mol}$ of the intermediate obtained above was taken in round bottom flask and dissolved in $50 \mathrm{ml}$ of ethanol. Different anillines $(0.001 \mathrm{~mol})$ were added to the flask and reaction mixture was refluxed for $8-10 \mathrm{~h}$ at $40^{\circ} \mathrm{C}$. Completion of reaction 


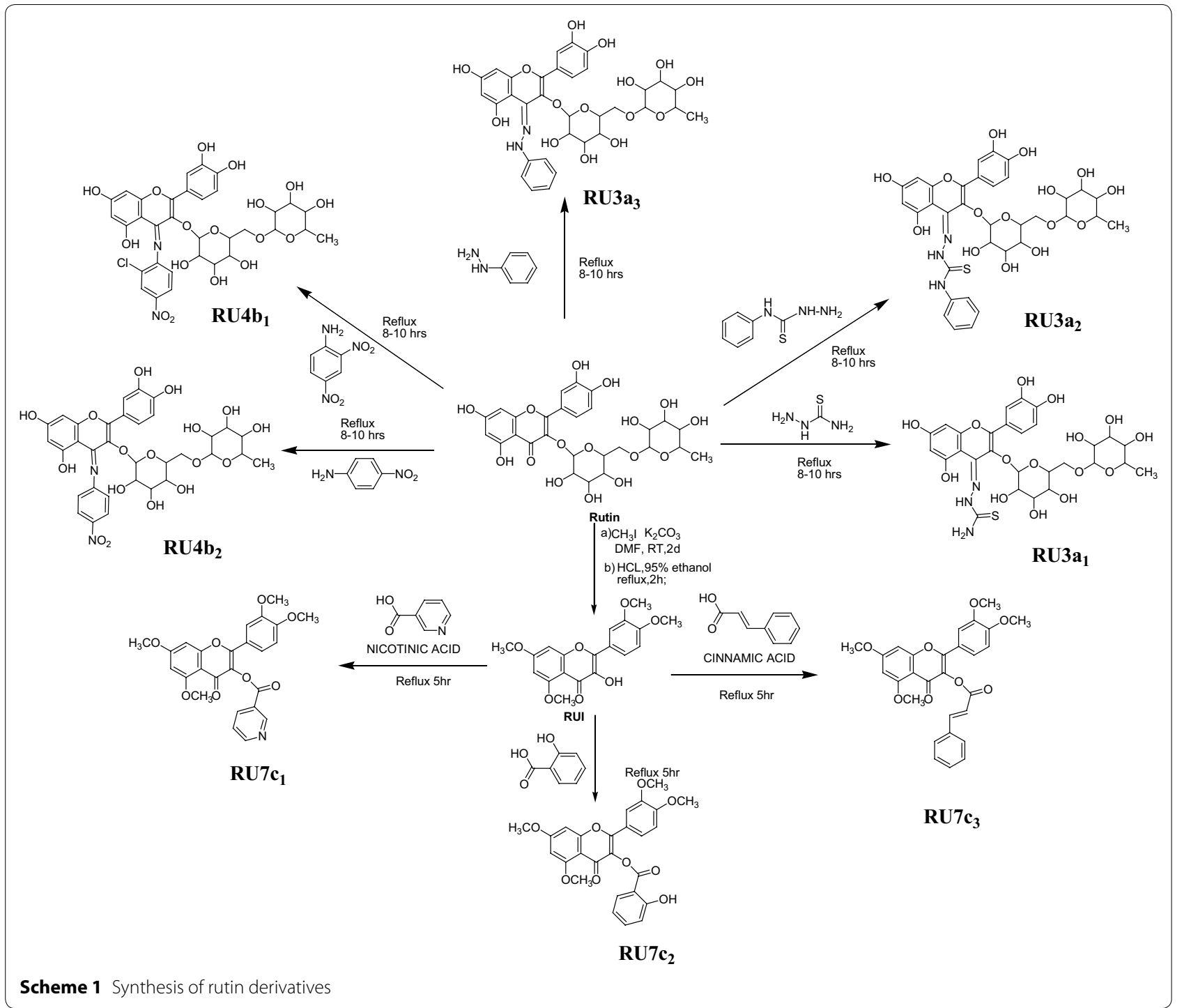

was monitored by TLC. The product thus obtained was filtered and filtrate was concentrated to obtain the final product. The final product was recrystallised to obtain the pure compound.

(C) General procedure for synthesis of methylated rutin derivatives $R U 7 c_{(1-3)}$

Rutin was methylated by methyl sulphate in presence of potassium carbonate and dimethyl formamide by stirring along with reflux at $40^{\circ} \mathrm{C}$ for $48 \mathrm{~h}$ to generate tetramethylated rutin. Acidolysis of above was done to obtain the intermediate compound (RUI) by refluxing it with $\mathrm{HCl}$ and $95 \%$ ethanol for $4 \mathrm{~h}$. The intermediate compound (RUI) was then refluxed with different phenolic acid to obtain their ester derivatives.
Spectral data $\mathrm{RU}_{3} \mathrm{a}_{1}$ yield $69.6 \% \mathrm{R}_{\mathrm{f}} 0.6 \quad$ [Mobile Phase for TLC-Methanol:Glacial acetic acid:Formic acid:Water (3:2.9:0.8:0.5)] M.pt. (231-232) IR (KBR pellets) $\mathrm{cm}^{-1}$ 1) 3222 (O-H str., Ar), 1609 (C=N str.), 1501 (C=C str.), $1206\left(\mathrm{O}-\mathrm{CH}_{3}\right), 1128$ (C=S Str.) ${ }^{1} \mathrm{H}$ NMR $\left(400 \mathrm{MHz}, \mathrm{DMSO}-d_{6}\right) \delta 7.81(\mathrm{dd}, J=7.5,1.5 \mathrm{~Hz}, 1 \mathrm{H})$, $7.59(\mathrm{~d}, J=1.5 \mathrm{~Hz}, 1 \mathrm{H}), 6.82(\mathrm{~d}, J=7.5 \mathrm{~Hz}, 1 \mathrm{H}), 6.48$ $(\mathrm{dd}, J=15.0,1.5 \mathrm{~Hz}, 2 \mathrm{H}), 6.28(\mathrm{t}, J=7.0 \mathrm{~Hz}, 1 \mathrm{H}), 4.13$ $(\mathrm{t}, J=7.0 \mathrm{~Hz}, 1 \mathrm{H}), 3.89-3.81(\mathrm{~m}, 3 \mathrm{H}), 3.71(\mathrm{dd}, J=12.4$, $6.9 \mathrm{~Hz}, 1 \mathrm{H}), 3.67-3.54(\mathrm{~m}, 3 \mathrm{H}), 2.32(\mathrm{dt}, J=12.4,7.0 \mathrm{~Hz}$, $1 \mathrm{H}), 2.28-2.16(\mathrm{~m}, 2 \mathrm{H}), 2.06-2.04(\mathrm{~m}, 1 \mathrm{H}), 1.97-1.92$ (m, 2H), 1.74-1.66 (m, 2H). ${ }^{13} \mathrm{C}$ NMR (100 MHz, Chloroform- $d) \delta 180.16,163.73,155.81,154.70,152.34,148.70$, $145.50,133.79,133.45,120.73,120.41,115.79,115.09$, 102.38, 99.59, 99.00, 91.11, 80.48, 73.58, 73.26, 72.40, 71.83 (d, $J=10.5 \mathrm{~Hz}$ ), 66.02, 40.22, 37.43, 28.26, 26.90. 
$\mathrm{m} / \mathrm{z}$ found for $\mathrm{C}_{28} \mathrm{H}_{33} \mathrm{~N}_{3} \mathrm{O}_{15} \mathrm{~S}: 683\left(\mathrm{M}^{+}\right) 687(\mathrm{M}+1)^{+}$. Anal calcd for $\mathrm{C}_{28} \mathrm{H}_{33} \mathrm{~N}_{3} \mathrm{O}_{15} \mathrm{~S}$ : C, 52.91; $\mathrm{H}, 5.23 ; \mathrm{N}, 6.61$; O, 35.20; S, 5.04 Found: C, 52.93; H, 5.21; N, 6.60; O, 35.19; S, 5.06 .

$\mathrm{RU}_{3}$ yield $72.5 \% \mathrm{R}_{\mathrm{f}} 0.7$ [Mobile Phase for TLCMethanol:Glacial acetic acid:Formic acid:Water (3:2.9:0.8:0.5)] M.pt. (255-257) IR (KBR pellets) $\mathrm{cm}^{-1}$ ) 3468 (O-H str., Ar), 1639 ( $\mathrm{C}=\mathrm{N}$ str.), 1596 (C=C str.), $1218\left(\mathrm{O}-\mathrm{CH}_{3}\right), 1150(\mathrm{C}=\mathrm{S}$ Str. $){ }^{1} \mathrm{H}$ NMR $(400 \mathrm{MHz}$, DMSO- $\left.d_{6}\right) \delta 7.78-7.60(\mathrm{~m}, 3 \mathrm{H}), 7.49(\mathrm{~d}, J=1.5 \mathrm{~Hz}, 1 \mathrm{H})$, 7.39-7.29 (m, 2H), 7.10-7.01 (m, 1H), $6.86(\mathrm{~d}, J=7.5 \mathrm{~Hz}$, $1 \mathrm{H}), 6.52(\mathrm{dd}, J=15.0,1.5 \mathrm{~Hz}, 2 \mathrm{H}), 6.24(\mathrm{t}, J=7.0 \mathrm{~Hz}$, $1 \mathrm{H}), 4.04(\mathrm{t}, J=7.0 \mathrm{~Hz}, 1 \mathrm{H}), 3.98-3.88(\mathrm{~m}, 3 \mathrm{H}), 3.78(\mathrm{dd}$, $J=12.4,6.9 \mathrm{~Hz}, 1 \mathrm{H}), 3.68-3.64(\mathrm{~m}, 3 \mathrm{H}), 2.28(\mathrm{dt}, J=12.4$, $7.0 \mathrm{~Hz}, 1 \mathrm{H}), 2.14-2.11(\mathrm{~m}, 2 \mathrm{H}), 2.09-2.06(\mathrm{~m}, 1 \mathrm{H}), 1.87-$ $1.84(\mathrm{~m}, 2 \mathrm{H}), 1.74-1.71(\mathrm{~m}, 2 \mathrm{H}) .{ }^{13} \mathrm{C}$ NMR $(100 \mathrm{MHz}$, Chloroform-d) $\delta 174.93,164.50,160.96,155.78,150.30$, $148.16,145.55,139.23,130.44,128.67,124.46,123.85$, $123.09,122.39,121.81,116.06,115.83,103.40,99.09$, 97.71, 95.05, 82.37, $73.06(\mathrm{~d}, J=19.1 \mathrm{~Hz}), 72.87(\mathrm{~d}$, $J=12.2 \mathrm{~Hz}), 72.47,72.35,71.92,65.19,41.10,38.86$, 29.40, 27.86. $\mathrm{m} / \mathrm{z}$ found for $\mathrm{C}_{34} \mathrm{H}_{37} \mathrm{~N}_{3} \mathrm{O}_{15} \mathrm{~S}: 759\left(\mathrm{M}^{+}\right) 760$ $(\mathrm{M}+1)^{+}$. Anal calcd for $\mathrm{C}_{34} \mathrm{H}_{37} \mathrm{~N}_{3} \mathrm{O}_{15} \mathrm{~S}$ : C, 53.75; H, 4.91; N, 5.53; O, 31.59; S, 4.22. Found: C, C, 53.77; H, 4.93; N, 5.56; O, 31.59; S, 4.24 .

RUT3a $_{3}$ yield $61 \% \mathrm{R}_{\mathrm{f}} 0.6$ [Mobile Phase for TLCMethanol:Glacial acetic acid:Formic acid:Water (3:2.9:0.8:0.5)] M.pt. (235-237) IR (KBR pellets) $\mathrm{cm}^{-1}$ ) 3475 (O-H str., Ar), 1641 (C=N str.), 1580 ( $\mathrm{C}=\mathrm{C}$ str.), $1220\left(\mathrm{O}-\mathrm{CH}_{3}\right), 1155$ (C=S Str.) ${ }^{1} \mathrm{H}$ NMR $(400 \mathrm{MHz}$, DMSO- $\left.d_{6}\right) \delta 7.70(\mathrm{dd}, J=7.5,1.5 \mathrm{~Hz}, 1 \mathrm{H}), 7.56(\mathrm{~d}$, $J=1.5 \mathrm{~Hz}, 1 \mathrm{H}), 7.46-7.38(\mathrm{~m}, 2 \mathrm{H}), 7.32-7.23(\mathrm{~m}, 2 \mathrm{H})$, $7.07-6.98(\mathrm{~m}, 1 \mathrm{H}), 6.89(\mathrm{~d}, J=7.5 \mathrm{~Hz}, 1 \mathrm{H}), 6.35(\mathrm{dd}$, $J=15.0,1.5 \mathrm{~Hz}, 2 \mathrm{H}), 6.19(\mathrm{t}, J=7.0 \mathrm{~Hz}, 1 \mathrm{H}), 4.09(\mathrm{t}$, $J=7.0 \mathrm{~Hz}, 1 \mathrm{H}), 4.02-3.88(\mathrm{~m}, 3 \mathrm{H}), 3.68(\mathrm{dd}, J=12.4$, $6.9 \mathrm{~Hz}, 1 \mathrm{H}), 3.66-3.54(\mathrm{~m}, 3 \mathrm{H}), 2.33(\mathrm{dt}, J=12.4,7.0 \mathrm{~Hz}$, $1 \mathrm{H}), 2.21-2.19(\mathrm{~m}, 2 \mathrm{H}), 1.96-1.88(\mathrm{~m}, 2 \mathrm{H}), 1.87-1.85(\mathrm{~m}$, $2 \mathrm{H})$ (Additional file 1). ${ }^{13} \mathrm{C}$ NMR $(100 \mathrm{MHz}$, Chloroformd) $\delta 164.50,160.96,155.78,150.30,148.16,145.55,143.60$, $132.14,129.50,124.46,122.39,121.81,121.19,118.32$, 116.06, 115.83, 104.75, 94.15, 93.97, 91.01, 83.98, 79.41 $(\mathrm{d}, J=19.1 \mathrm{~Hz}), 78.77$ (d, $J=12.2 \mathrm{~Hz}), 77.09,73.82,68.48$, 42.85, 37.51, 23.82, 23.17. $\mathrm{m} / \mathrm{z}$ found for $\mathrm{C}_{33} \mathrm{H}_{36} \mathrm{~N}_{2} \mathrm{O}_{15}$ : $700\left(\mathrm{M}^{+}\right) 701(\mathrm{M}+1)^{+}$. Anal calcd for $\mathrm{C}_{33} \mathrm{H}_{36} \mathrm{~N}_{2} \mathrm{O}_{15}: \mathrm{C}$, 56.57; H, 5.18; N, 4.00; O, 34.25. Found: C, 56.58; H, 5.20; $\mathrm{N}, 4.00 ; \mathrm{O}, 34.27$.

RU4b $b_{1}$ yield $74.3 \% R_{f} 0.6$ [Mobile Phase for TLCMethanol:Glacial acetic acid:Formic acid:Water (3:2.9:0.8:0.5)] M.pt. (259-260) IR (KBR pellets) $\mathrm{cm}^{-1} 1$ ) 1725 ( $\mathrm{C}=\mathrm{O}$ str.), 1631 ( $\mathrm{C}=\mathrm{N}$ str.), 1603 ( $\mathrm{C}=\mathrm{C}$ str.), 1234 $\left(\mathrm{O}-\mathrm{CH}_{3}\right), 1268(\mathrm{C}-\mathrm{O} \text { str., ester })^{1} \mathrm{H}$ NMR $(400 \mathrm{MHz}$, DMSO- $\left.d_{6}\right) \delta 8.38(\mathrm{~d}, J=1.5 \mathrm{~Hz}, 1 \mathrm{H}), 8.15(\mathrm{dd}, J=7.5$, $1.5 \mathrm{~Hz}, 1 \mathrm{H}), 7.69(\mathrm{dd}, J=7.5,1.5 \mathrm{~Hz}, 1 \mathrm{H}), 7.2(\mathrm{~d}$,
$J=1.5 \mathrm{~Hz}, 1 \mathrm{H}), 7.40(\mathrm{~d}, J=7.5 \mathrm{~Hz}, 1 \mathrm{H}), 6.81(\mathrm{~d}, J=7.5 \mathrm{~Hz}$, $1 \mathrm{H}), 6.47(\mathrm{dd}, J=10.8,1.5 \mathrm{~Hz}, 2 \mathrm{H}), 6.22(\mathrm{t}, J=7.0 \mathrm{~Hz}$, $1 \mathrm{H}), 4.11(\mathrm{t}, J=7.0 \mathrm{~Hz}, 1 \mathrm{H}), 3.98-3.90(\mathrm{~m}, 3 \mathrm{H}), 3.79(\mathrm{dd}$, $J=12.4,6.9 \mathrm{~Hz}, 1 \mathrm{H}), 3.71-3.61(\mathrm{~m}, 3 \mathrm{H}), 2.42(\mathrm{dt}, J=12.4$, $7.0 \mathrm{~Hz}, 1 \mathrm{H}), 2.39-2.31(\mathrm{~m}, 2 \mathrm{H}), 2.29-2.28(\mathrm{~m}, 1 \mathrm{H})$, 1.87-1.77 (m, 2H). ${ }^{13} \mathrm{C}$ NMR (100 MHz, Chloroform- $d$ ) $\delta$ 169.14, 168.95, 168.11, 166.86, 150.94, 144.52, 144.24, $142.37,140.47,131.18,128.56,125.41,123.81,122.54$ (d, $J=14.8 \mathrm{~Hz}), 121.81,113.64,113.17,106.71,97.09,96.89$, 93.98, 82.37, 75.79 (d, $J=19.1 \mathrm{~Hz}), 73.17$ (d, $J=12.2 \mathrm{~Hz}$ ), 73.06, 72.69, 71.01, 65.19, 41.10, 38.86, 28.85, 27.44. m/z found for $\mathrm{H}_{33} \mathrm{ClN}_{2} \mathrm{O}_{17}: 764\left(\mathrm{M}^{+}\right) 766(\mathrm{M}+2)^{+}$. Anal calcd for $\mathrm{C}_{33} \mathrm{H}_{33} \mathrm{ClN}_{2} \mathrm{O}_{17}$ : C, 51.81; $\mathrm{H}, 4.35 ; \mathrm{Cl}, 4.63 ; \mathrm{N}, 3.66$; O, 35.55. Found: C, 51.83; H, 4.36; Cl, 4.65; N, 3.64; O, 35.53 .

RU $4 b_{2}$ yield $83.5 \% R_{f} 0.8$ [Mobile Phase for TLCMethanol:Glacial acetic acid:Formic acid:Water (3:2.9:0.8:0.5)] M.pt. (253-254) IR (KBR pellets) $\mathrm{cm}^{-1} 1$ ) 1785 ( $\mathrm{C}=\mathrm{O}$ str.), 1637 ( $\mathrm{C}=\mathrm{N}$ str.), 1561 ( $\mathrm{C}=\mathrm{C}$ str.), 1258 $\left(\mathrm{O}-\mathrm{CH}_{3}\right), 1234\left(\mathrm{C}-\mathrm{O}\right.$ str., ester) ${ }^{1} \mathrm{H}$ NMR $(400 \mathrm{MHz}$, DMSO- $\left.d_{6}\right) \delta 8.21-8.14(\mathrm{~m}, 2 \mathrm{H}), 7.79(\mathrm{dd}, J=7.5,1.5 \mathrm{~Hz}$, $1 \mathrm{H}), 7.59$ (d, J=1.5 Hz, 1H), 7.32-7.25 (m, 2H), 6.75 (d, $J=7.5 \mathrm{~Hz}, 1 \mathrm{H}), 6.44(\mathrm{dd}, J=14.1,1.5 \mathrm{~Hz}, 2 \mathrm{H}), 6.27(\mathrm{t}$, $J=7.0 \mathrm{~Hz}, 1 \mathrm{H}), 4.15(\mathrm{t}, J=7.0 \mathrm{~Hz}, 1 \mathrm{H}), 3.98-3.95(\mathrm{~m}$, $3 \mathrm{H}), 3.88(\mathrm{dd}, J=12.4,6.9 \mathrm{~Hz}, 1 \mathrm{H}), 3.67-3.55(\mathrm{~m}, 3 \mathrm{H})$, $2.22(\mathrm{dt}, J=12.4,7.0 \mathrm{~Hz}, 1 \mathrm{H}), 2.14-2.11(\mathrm{~m}, 2 \mathrm{H}), 2.09$ $2.06(\mathrm{~m}, 1 \mathrm{H}), 1.76-1.73(\mathrm{~m}, 2 \mathrm{H}), 1.67-1.55(\mathrm{~m}, 2 \mathrm{H})$.

${ }^{13} \mathrm{C}$ NMR $(100 \mathrm{MHz}$, Chloroform-d) $\delta 173.89,164.58$, $163.50,158.34,152.36,151.92,148.16,146.53,145.55$, $128.56,125.27,124.36,122.39,121.81,116.06,115.83$, 108.81, 93.06, 97.81, 90.53, 82.19, $73.80(\mathrm{~d}, J=19.1 \mathrm{~Hz})$, $72.67(\mathrm{~d}, J=12.2 \mathrm{~Hz}), 72.36,72.12,71.08,64.86,42.81$, $36.15,28.55,26.98 . \mathrm{m} / \mathrm{z}$ found for $\mathrm{C}_{33} \mathrm{H}_{34} \mathrm{~N}_{2} \mathrm{O}_{17}: 730\left(\mathrm{M}^{+}\right)$ $731(\mathrm{M}+1)^{+}$. Anal calcd for $\mathrm{C}_{33} \mathrm{H}_{34} \mathrm{~N}_{2} \mathrm{O}_{17}: \mathrm{C}, 54.25 ; \mathrm{H}$, 4.69; N, 3.83; O, 37.23. Found: C, 54.27; H, 4.70; N, 3.85; $\mathrm{O}, 37.25$.

RU7C $_{1}$ yield $83.5 \% \mathrm{R}_{\mathrm{f}} 0.8$ [Mobile Phase for TLCMethanol:Glacial acetic acid:Formic acid:Water (3:2.9:0.8:0.5)] M.pt. (189-190) IR (KBR pellets) $\mathrm{cm}^{-1} 1$ ) 1715 ( $\mathrm{C}=\mathrm{O}$ str.), 1627 ( $\mathrm{C}=\mathrm{N}$ str.), 1607 ( $\mathrm{C}=\mathrm{C}$ str.), 1234 $\left(\mathrm{O}-\mathrm{CH}_{3}\right), 11,944\left(\mathrm{C}-\mathrm{O}\right.$ str., ester) ${ }^{1} \mathrm{H}$ NMR $(400 \mathrm{MHz}$, DMSO- $\left.d_{6}\right) \delta 9.11(\mathrm{~d}, J=1.5 \mathrm{~Hz}, 1 \mathrm{H}), 8.77-8.70(\mathrm{~m}, 1 \mathrm{H})$, $8.14(\mathrm{dt}, J=7.5,1.5 \mathrm{~Hz}, 1 \mathrm{H}), 7.92(\mathrm{dd}, J=7.5,1.5 \mathrm{~Hz}$, $1 \mathrm{H}), 7.68(\mathrm{~d}, J=1.5 \mathrm{~Hz}, 1 \mathrm{H}), 7.51(\mathrm{t}, J=7.5 \mathrm{~Hz}, 1 \mathrm{H})$, 6.93-6.83 (m, 2H), $6.23(\mathrm{~d}, J=1.5 \mathrm{~Hz}, 1 \mathrm{H}), 3.92(\mathrm{~s}, 3 \mathrm{H})$, $3.83(\mathrm{~d}, J=0.9 \mathrm{~Hz}, 6 \mathrm{H}), 3.76(\mathrm{~s}, 3 \mathrm{H}) .{ }^{13} \mathrm{C}$ NMR $(100 \mathrm{MHz}$, Chloroform- $d$ ) $\delta 174.99,164.48,164.18,160.33,157.96$, $156.60,153.53,151.74,150.80,149.32,138.25,128.95$, $123.72,123.22,122.87,122.65,113.70,112.82,107.81$, $95.68,93.25,56.20,55.88(\mathrm{~d}, J=2.6 \mathrm{~Hz}), 55.62 . \mathrm{m} / \mathrm{z}$ found for $\mathrm{C}_{25} \mathrm{H}_{21} \mathrm{NO}_{8}: 463\left(\mathrm{M}^{+}\right)$464 $(\mathrm{M}+1)^{+}$. Anal calcd for $\mathrm{C}_{25} \mathrm{H}_{21} \mathrm{NO}_{8}$ : C, 64.79; $\mathrm{H}, 4.57 ; \mathrm{N}, 3.02 ; \mathrm{O}, 27.62$. Found: $\mathrm{C}$, $64.80 ; \mathrm{H}, 4.58 ; \mathrm{N}, 3.00 ; \mathrm{O}, 27.60$. 
$\mathrm{RU7C}_{2}$ yield $62.5 \% \mathrm{R}_{\mathrm{f}} 0.6$ [Mobile Phase for TLCMethanol:Glacial acetic acid:Formic acid:Water (3:2.9:0.8:0.5)] M.pt. (186-188) IR (KBR pellets) $\mathrm{cm}^{-1} 1$ ) 1764 ( $\mathrm{C}=\mathrm{O}$ str.), 1619 ( $\mathrm{C}=\mathrm{N}$ str.), 1595 ( $\mathrm{C}=\mathrm{C}$ str.), 1277 $\left(\mathrm{O}-\mathrm{CH}_{3}\right), 1214\left(\mathrm{C}-\mathrm{O}\right.$ str., ester) ${ }^{1} \mathrm{H}$ NMR $(400 \mathrm{MHz}$, DMSO- $d_{6}$ ) $\delta 7.91$ (ddd, $J=7.5,6.5,1.5 \mathrm{~Hz}, 2 \mathrm{H}$ ), 7.67 (d, $J=1.5 \mathrm{~Hz}, 1 \mathrm{H}), 7.47$ (td, $J=7.5,1.5 \mathrm{~Hz}, 1 \mathrm{H}), 7.09$ $(\mathrm{td}, J=7.5,1.5 \mathrm{~Hz}, 1 \mathrm{H}), 6.97-6.88(\mathrm{~m}, 2 \mathrm{H}), 6.86(\mathrm{~d}$, $J=1.5 \mathrm{~Hz}, 1 \mathrm{H}), 6.28(\mathrm{~d}, J=1.5 \mathrm{~Hz}, 1 \mathrm{H}), 3.97(\mathrm{~s}, 3 \mathrm{H}), 3.80$ $(\mathrm{d}, J=0.7 \mathrm{~Hz}, 6 \mathrm{H}), 3.67(\mathrm{~s}, 3 \mathrm{H}) .{ }^{13} \mathrm{C}$ NMR $(100 \mathrm{MHz}$, Chloroform-d) $\delta 171.85,168.95,167.67,165.22,158.95$, $157.67,148.53,146.92,133.72,131.16,128.84,124.78$, $124.78,123.22,122.87,116.52,113.70,108.53,104.92$, $92.81,90.38,53.06,52.81,52.76(\mathrm{~d}, J=2.6 \mathrm{~Hz}), 51.65 . \mathrm{m} / \mathrm{z}$ found for $\mathrm{C}_{26} \mathrm{H}_{22} \mathrm{O}_{9}: 478\left(\mathrm{M}^{+}\right) 479(\mathrm{M}+1)^{+}$. Anal calcd for $\mathrm{C}_{26} \mathrm{H}_{22} \mathrm{O}_{9}$ : C, 65.27; H, 4.63; O, 30.10. Found: C, 65.27; $\mathrm{H}, 4.63$; O, 30.10 .

RU7C $_{3}$ yield $71 \% \mathrm{R}_{\mathrm{f}} 0.7$ [Mobile Phase for TLCMethanol:Glacial acetic acid:Formic acid:Water (3:2.9:0.8:0.5)] M.pt. (165-166) IR (KBR pellets) $\mathrm{cm}^{-1} 1$ ) 1710 ( $\mathrm{C}=\mathrm{O}$ str.), 1637 ( $\mathrm{C}=\mathrm{N}$ str.), 1596 ( $\mathrm{C}=\mathrm{C}$ str.), 1258 $\left(\mathrm{O}-\mathrm{CH}_{3}\right), 1194\left(\mathrm{C}-\mathrm{O}\right.$ str., ester) ${ }^{1} \mathrm{H}$ NMR $(400 \mathrm{MHz}$, DMSO-d $\left.{ }_{6}\right) \delta 7.98(\mathrm{dd}, J=7.5,1.5 \mathrm{~Hz}, 1 \mathrm{H}), 7.76(\mathrm{~d}$, $J=1.5 \mathrm{~Hz}, 1 \mathrm{H}), 7.30-7.20(\mathrm{~m}, 5 \mathrm{H}), 6.91-6.86(\mathrm{~m}, 2 \mathrm{H})$, $6.23(\mathrm{~d}, J=1.5 \mathrm{~Hz}, 1 \mathrm{H}), 3.93(\mathrm{~s}, 3 \mathrm{H}), 3.88(\mathrm{~d}, J=0.9 \mathrm{~Hz}$, $6 \mathrm{H}), 3.69(\mathrm{~s}, 3 \mathrm{H}), 2.93-2.84(\mathrm{~m}, 2 \mathrm{H}), 2.73(\mathrm{td}, J=7.0$, $0.8 \mathrm{~Hz}, 2 \mathrm{H}) .{ }^{13} \mathrm{C}$ NMR $(100 \mathrm{MHz}$, Chloroform-d $) \delta$ $175.20,170.26,164.48,160.33,157.96,156.95,150.80$, $149.32,139.89,128.47-128.31(\mathrm{~m}), 126.14,123.22$, $122.87,113.70,112.82,107.81,99.41,98.77,53.17,53.06$ (d, $J=2.6 \mathrm{~Hz}$ ), 52.69, 51.86, 34.56, 30.26. $\mathrm{m} / \mathrm{z}$ found for $\mathrm{C}_{28} \mathrm{H}_{24} \mathrm{O}_{8}: 488\left(\mathrm{M}^{+}\right) 489(\mathrm{M}+1)^{+}$. Anal calcd for $\mathrm{C}_{28} \mathrm{H}_{24} \mathrm{O}_{8}: \mathrm{C}, 68.85 ; \mathrm{H}, 4.95 ; \mathrm{O}, 26.20$. Found: $\mathrm{C}, 68.87 ; \mathrm{H}$, 4.90; O, 26.20.

\section{Evaluation of biological activity In vitro evaluation of xanthine oxidase inhibitory activity}

The method opted to evaluate the inhibitory potential of rutin derivatives was a modified protocol of Sigma, done by UV-spectrophotometric method by using xanthine oxidase activity assay kit purchased from sigma (MAK078, sigma-aldrich.co, USA). The colorimetric product obtained in the form of hydrogen peroxide generated during the oxidation of $\mathrm{XO}$ was determined by a coupled enzyme technique, measured at $570 \mathrm{~nm}$ in a 96-well plate, using the plate reader $\mathrm{EPOCH}^{\mathrm{TM}}$ "MICROPLATE READER (BIOTEK).one unit of XO is defined as the amount of enzyme that catalyzes the oxidation of xanthine substrate, yielding $1.0 \mu \mathrm{mol}$ of uric acid and hydrogen peroxide per minute at $25{ }^{\circ} \mathrm{C}$. Reagents used were $44 \mu \mathrm{L}$ of xanthine oxidase assay buffer, $2 \mu \mathrm{l}$ xanthine substrate solution and $2 \mu \mathrm{l}$ of Xanthine Oxidase enzyme solution. All the solutions mentioned above were mixed to prepare reaction mixture. The different concentrations of synthesized derivatives having final volume $50 \mu \mathrm{l}$ were prepared in dimethyl sulfoxide (DMSO) and added to 96 well plate. To each well $50 \mu \mathrm{l}$ of reaction mix was added and mixed well. After 2-3 min initial measurement was taken. The plates were incubated at $25{ }^{\circ} \mathrm{C}$ taking measurements at every $5 \mathrm{~min}$. Allopurinol served as positive control. Absorbance at different time intervals was noted for further statistical analysis.

\section{In vitro evaluation of antioxidant activity by DPPH method}

The antioxidant potential of rutin derivatives was performed by DPPH method evaluated in the form of $\mathrm{IC}_{50}$ estimated using the ELISA plate reader $\mathrm{EPOCH}^{\mathrm{TM}}$ "MICROPLATE READER (BIOTEK). This method opted for evaluation of free radical scavenging activity of DPPH was based on modified procedure described by Dhiman et al. [36]. The tested compounds were prepared in methanolic solution and reacted with methanolic solution of $\mathrm{DPPH}$ at $37{ }^{\circ} \mathrm{C}$. The reaction mixture was prepared in 96-well plate by adding $50 \mu \mathrm{L}$ of sample, $50 \mu \mathrm{l}$ of methanol and $50 \mu \mathrm{l}$ of DPPH solution prepared in $0.1 \mathrm{mM}$ methanol. The mechanism of action of DPPH assay was based on the fact that DPPH radical get reduced during its reaction with an antioxidant compound and results in changes of color (from deep violet to light yellow). The absorbance was read at $517 \mathrm{~nm}$ for $30 \mathrm{~min}$ at an interval of 5 min of using ELISA microplate reader. The mixture of methanol $(5.0 \mathrm{ml})$ and tested compounds $(0.2 \mathrm{ml})$ serve as blank. Ascorbic acid served as positive control.

\section{Hydrogen peroxide scavenging $\left(\mathrm{H}_{2} \mathrm{O}_{2}\right)$ assay}

To compare and best evaluate the antioxidant potential of newly synthesized rutin derivatives, hydrogen peroxide assay was performed by the method described by Patel et al. [37] with some modifications. The solution of $\mathrm{H}_{2} \mathrm{O}_{2}(100 \mathrm{mM})$ was prepared via adding up different concentrations of synthesized derivatives ranging from 5 to $80 \mu \mathrm{g} / \mathrm{ml}$ to $\mathrm{H}_{2} \mathrm{O}_{2}$ solution ( $2 \mathrm{ml}$ ), prepared in $20 \mathrm{mM}$ phosphate buffer of $\mathrm{pH}$ 7.4. Finally, the absorbance of $\mathrm{H}_{2} \mathrm{O}_{2}$ was measured at $230 \mathrm{~nm}$ after incubating for 10 min next to a blank reading of phosphate buffer without $\mathrm{H}_{2} \mathrm{O}_{2}$. For every measurement, a fresh reading of blank was taken to carry out background correction. For control sample containing $\mathrm{H}_{2} \mathrm{O}_{2}$ was scanned for absorbance at $230 \mathrm{~nm}$. Results calculated as percentage of hydrogen peroxide inhibition was estimated by the formula $\left[\left(A_{b}-A_{t}\right) / A_{0}\right] \times 100$, where $A_{b}$ is the absorbance of the control and $A_{t}$ is the absorbance of compounds/ standard taken as L-ascorbic acid $(5-80 \mu \mathrm{g} / \mathrm{ml})$ are shown in Table 5. 
Table 1 ADMET data of natural ligands calculated using Qik Prop simulation

\begin{tabular}{|c|c|c|c|c|c|c|c|c|c|c|}
\hline Compound & QPlogPo/w & QPlogS & QPlogHERG & QPPCaco & QPlogBB & QPPMDCK & QPlogKp & QPlogKhsa & $\begin{array}{l}\text { Human oral } \\
\text { absorption }\end{array}$ & $\begin{array}{l}\text { Percent human } \\
\text { oral absorption }\end{array}$ \\
\hline $\mathrm{RU}_{3} \mathrm{a}_{1}$ & -1.084 & -3.257 & -5.488 & 511.672 & -2.173 & 625.905 & -6.818 & -0.902 & 2 & 81 \\
\hline $\mathrm{RU}_{3} \mathrm{a}_{2}$ & 0.866 & -4.593 & -7.183 & 605.947 & -1.139 & 853.322 & -4.846 & -0.635 & 2 & 77 \\
\hline $\mathrm{RU}_{3} \mathrm{a}_{3}$ & 0.444 & -2.809 & -5.496 & 758.912 & -1.381 & 793.01 & -4.796 & -0.58 & 3 & 76 \\
\hline$R \cup 4 b_{1}$ & -0.044 & -3.745 & -6.548 & 563.916 & -2.192 & 641.237 & -5.52 & -0.747 & 1 & 60 \\
\hline$R \cup 4 b_{2}$ & 0.407 & -4.15 & -6.511 & 941.594 & -2.757 & 730.468 & -6.278 & -0.533 & 1 & 50 \\
\hline$R \cup 7 c_{1}$ & 3.322 & -4.469 & -6.334 & 1460.431 & -0.726 & 744.963 & -1.477 & -0.218 & 3 & 100 \\
\hline $\mathrm{RUTC}_{2}$ & 4.878 & -5.717 & -6.59 & 2335.951 & -0.63 & 1237.701 & -0.774 & 0.383 & 3 & 100 \\
\hline $\mathrm{RUTC}_{3}$ & -0.334 & -3.885 & -6.168 & 743.251 & -1.271 & 971.012 & -6.276 & -0.735 & 2 & 50 \\
\hline Rutin & -0.28 & -2.94 & -5.166 & 827.655 & -3.378 & 682.554 & -5.639 & -0.703 & 1 & 30 \\
\hline Allopurinol & -1.365 & -2.932 & -0.839 & 569.551 & -3.6 & -570.702 & -6.890 & -0.986 & 2 & 50 \\
\hline
\end{tabular}

Descriptor standard range: QPlogPo/w, -2.0 to 6.5; QPlogS, -6.5 to 0.5 ; QPlogHERG, concern below -5 ; QPPCaco, <25 poor, > 500 great; QPlogBB, - 3.0 to 1.2; QPPMDCK, <25 poor, > 500 great; QPlogKp, -8.0 to -1.0 ; QPlogKhsa, -1.5 to 1.5; human oral absorption, 1, 2, or 3 for low, medium, or high; percent human oral absorption, $>80 \%$ is high

\section{ADMET studies}

The pharmacokinetic and pharmacological parameters of newly synthesized compounds were predicted with the help of Schrodinger suite. In-silico ADMET-related properties were computed using Qikprop application of Schrodinger software (Table 1). QikProp program generates set of physicochemically significant descriptors which further evaluates ADMET properties. The whole ADME-compliance score-drug-likeness parameter is used to predict the pharmacokinetic profiles of the ligands. This parameter determines the number of property descriptors calculated via QikProp which fall outside from the optimum range of values for $95 \%$ of noted drugs. Initially, all compound structures were neutralized before operated through Qikprop. The neutralizing step is crucial, as QikProp is unable to neutralize ligands in normal mode. Qikprop predicts both pharmacokinetically significant properties and physicochemically significant descriptors. It application run in normal mode which predicted $\mathrm{IC}_{50}$ value for blockage of HERG $\mathrm{K}+$ channels (log HERG), predicted apparent Caco-2 cell permeability in $\mathrm{nm} / \mathrm{s}$ (QPPCaco), brain/blood partition coefficient (QPlogBB), predicted skin permeability (QPlogKp), prediction of binding to human serum albumin (QPlogKhsa) and predicted apparent MadinDarby Canine Kidney (MDCK) cell permeability in $\mathrm{nm} / \mathrm{s}$ (QPPMDCK). Solubility of drug was predicted as octanol/ water partition coefficient (QPlogPo/w). Aqueous solubility of compound defined in terms of $\log \mathrm{S}\left(\mathrm{S}\right.$ in $\left.\mathrm{mol} \mathrm{dm}^{-3}\right)$ is the concentration of the solute in a saturated solution that is in equilibrium with the crystalline solid.

\section{Result and discussion} Molecular docking

To rationalize the structure activity relationship observed in this research and to foreknow the potential interaction
Table 2 Comparison of in vitro activity and molecular docking studies

\begin{tabular}{lcll}
\hline Compound & Docking score & $\begin{array}{l}\text { Binding } \\
\text { energy }[\Delta \mathbf{G} \\
(\mathbf{K J} / \mathbf{m o l})]\end{array}$ & $\mathbf{I C}_{\mathbf{5 0}}(\boldsymbol{\mu M})$ \\
\hline $\mathrm{RU3a}_{1}$ & -12.907 & -88.383 & $09.924 \pm 0.01$ \\
$\mathrm{RU3a}_{2}$ & -11.456 & -67.673 & $07.905 \pm 0.15$ \\
$\mathrm{RU3a}_{3}$ & -13.244 & -91.242 & $04.870 \pm 0.02$ \\
$\mathrm{RU}_{4} \mathrm{~b}_{1}$ & -11.591 & -60.323 & $15.037 \pm 0.01$ \\
$\mathrm{RU}_{4} \mathrm{~b}_{2}$ & -12.021 & -72.991 & $12.541 \pm 0.45$ \\
$\mathrm{RU7C}_{1}$ & -11.310 & -55.854 & $19.377 \pm 0.38$ \\
$\mathrm{RU7C}_{2}$ & -10.980 & -61.268 & $17.428 \pm 0.01$ \\
$\mathrm{RU7C}_{3}$ & 11.037 & 50.217 & $13.476 \pm 0.25$ \\
Rutin & -10.944 & -45.549 & $20.867 \pm 0.12$ \\
Allopurinol & -3.366 & -17.231 & $10.410 \pm 0.72$ \\
\hline
\end{tabular}

Italic values indicating standard drug

of the synthesized compounds with $\mathrm{XO}$, molecular simulation studies were carried out using Schrödinger suite (Schrödinger Release 2018-2, Schrödinger, LLC, New York, NY, 2018).The crystal structure of xanthine oxidase with PDB code 2E1Q was adopted for the docking calculations. Based on the docking score and binding energy calculation, top ranking derivatives were established and compared with the $\mathrm{IC}_{50}$ calculated from in vitro activity (Table 2). Important interactions were depicted as hydrophobic regions, hydrogen bonding, polar interactions and pi-pi bonding visualized in the active pocket of xanthine oxidase revealed through Site map application of Schrodinger suite. The derivatives having better docking scores than rutin were kept for further synthetic procedures and the remaining were discarded. To observe the binding interaction in detail, 3D poses of two most 


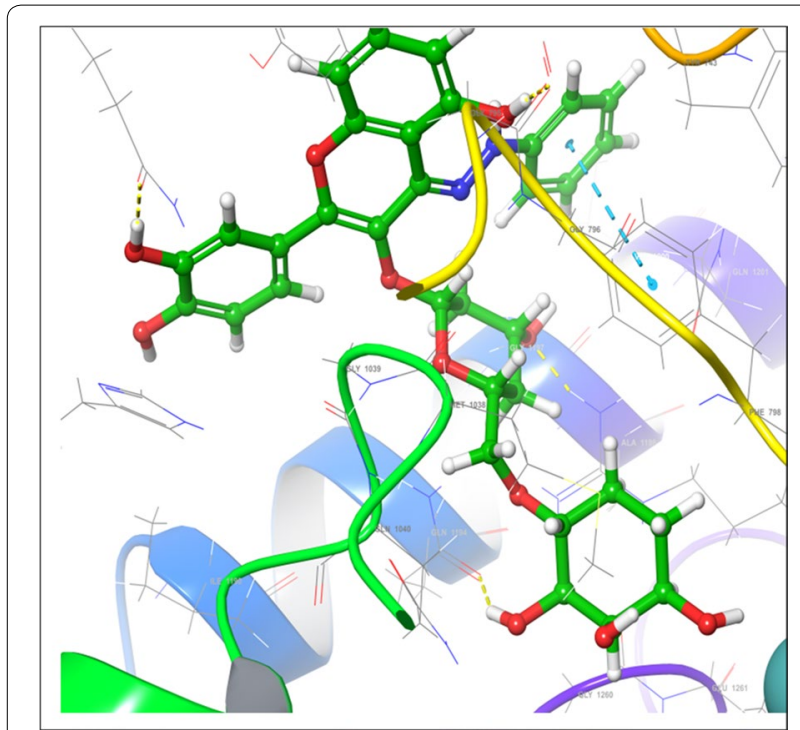

Fig. $23 \mathrm{D}$ pose of $\mathrm{RU}_{3} \mathrm{a}_{3}$ inside the binding pocket

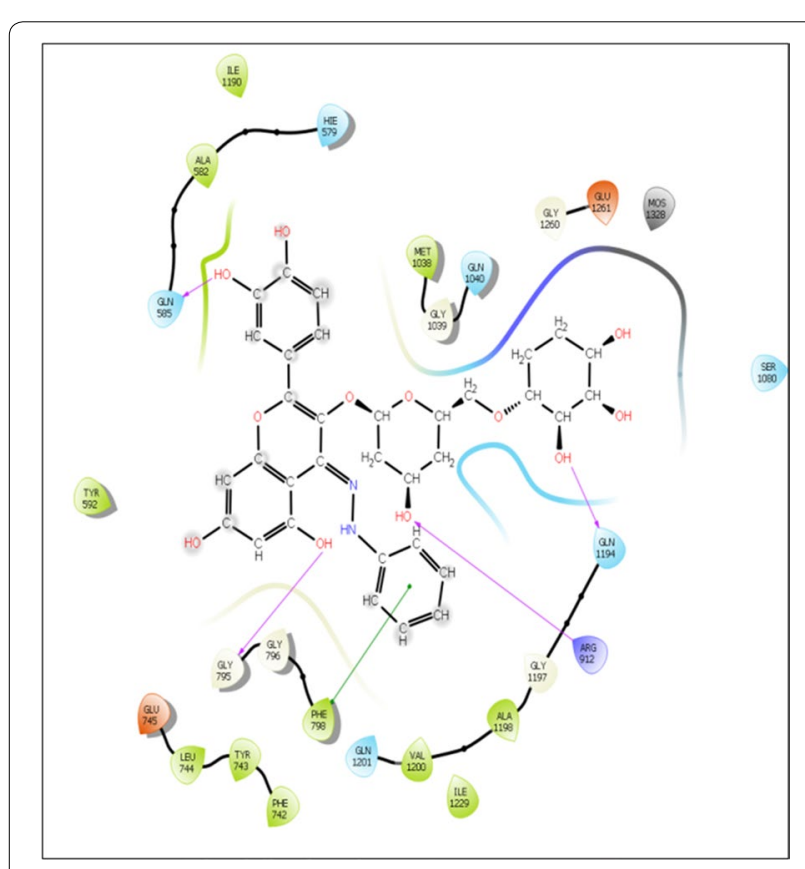

Fig. $32 \mathrm{D}$ pose of $\mathrm{RU}_{3} \mathrm{a}_{3}$ inside the binding pocket

active compounds RU3a $a_{3}$ and RU3a $a_{1}$ were visualized and compared with native rutin and standard drug Allopurinol. The residues of binding pocket involved in the interaction were reported as GLN 1194, ARG912, MET1038, GLN1040, PHE798 and SER1080. Similar binding cavity was observed by Li et al. during the docking analysis of newly synthesized non-purine XO inhibitors [38].

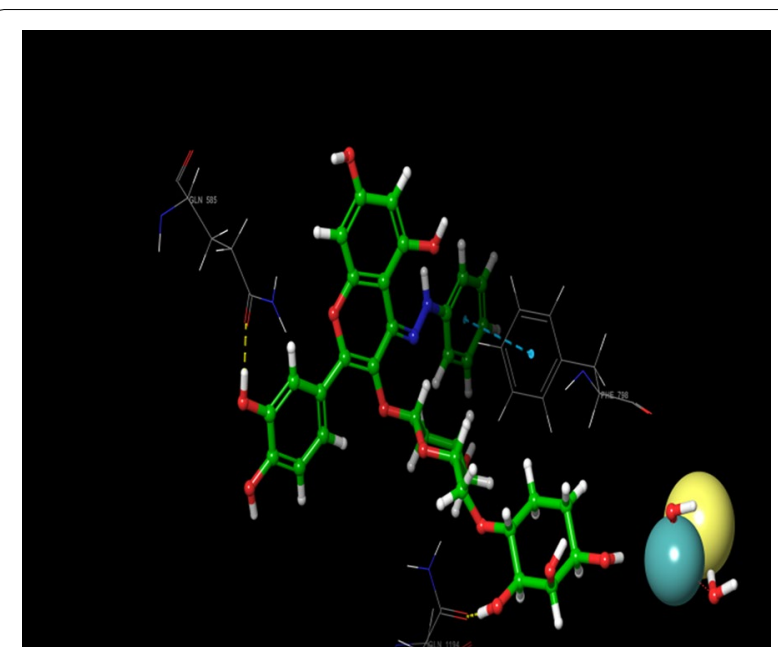

Fig. 4 3D pose of $R U 3 a_{3}$ showing hydrogen bonding (yellow) with GLN1 194, ARG 912, GLY795, GLN 585 and $\pi-\pi$ bonding (blue) with PHE798

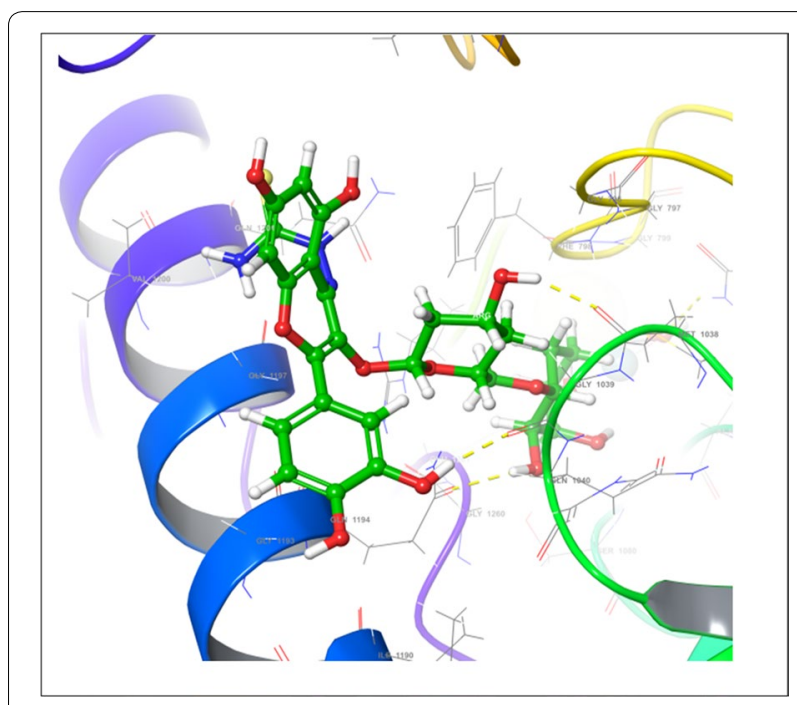

Fig. $53 \mathrm{D}$ pose of $\mathrm{RU} \mathrm{a}_{1}$ inside the binding pocket

Visual inspection of 3D poses of $\mathrm{RU}_{3} \mathrm{a}_{3}$ displayed a compact arrangement of polar and hydrophobic residues around the ligand forming a narrow passage in $\mathrm{XO}$ binding cavity with a docking score/binding score of -13.244 and binding energy $-91.242 \mathrm{~kJ} / \mathrm{mol}$. An interesting pi-pi bonding was observed between benzene ring of phenyl hydrazine and hydrophobic residue PHE 798 of active site (Figs. 1, 2, 3). Along with this a strong hydrogen bonding was observed between $\mathrm{OH}$ group of rutinoside and polar residue GLN 1194 and negatively charged ARG 912 (Fig. 4). Similarly ARG 912 was found essential in the study of Shen et al. during the comparison of curcumin 


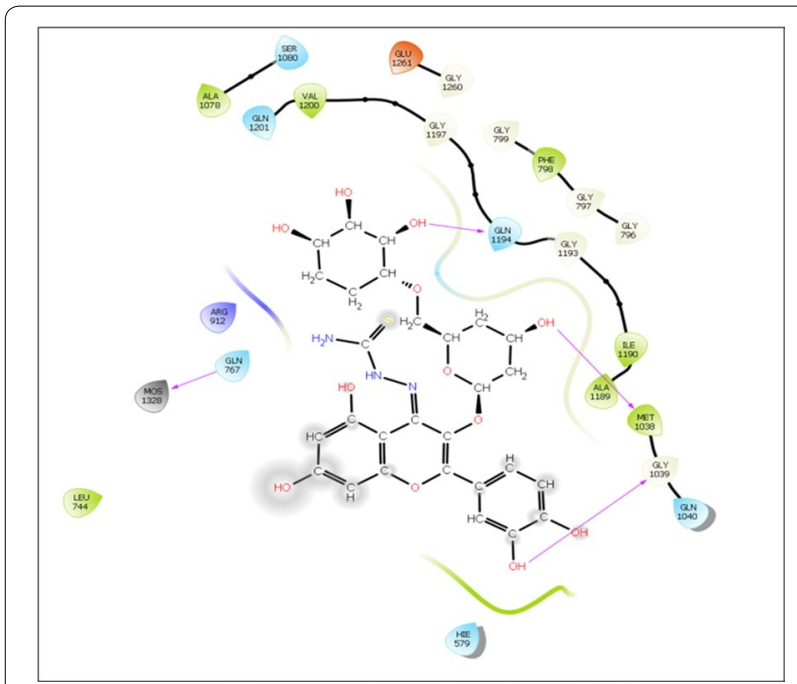

Fig. $62 \mathrm{D}$ pose of $\mathrm{RU}_{3} \mathrm{a}_{1}$ inside the binding pocket

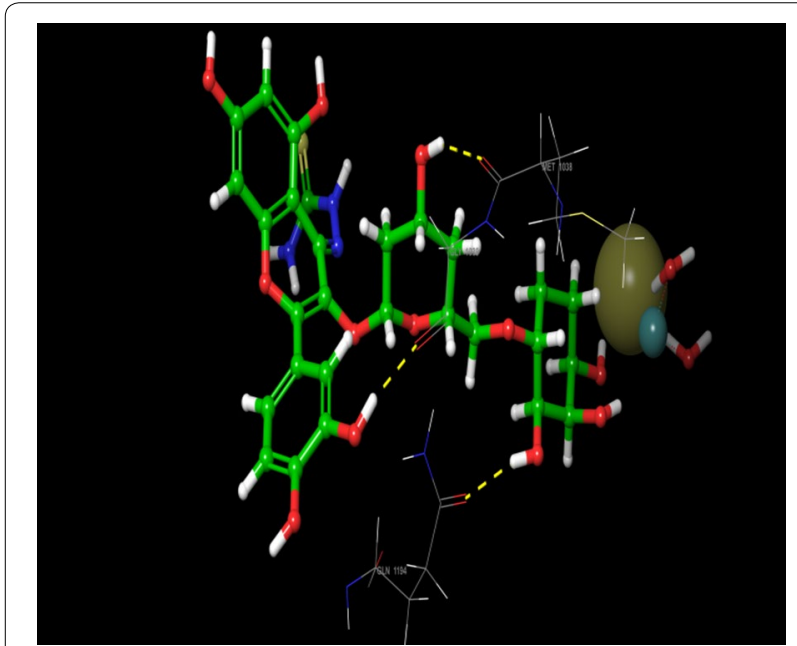

Fig. 7 3D pose of RU3a, showing hydrogen bonding with GLN 1194, MET1038 and GLY 1039

derivatives with quercetin and leuteolin [39]. Another hydrogen bonding was visualized between Chromene moiety and the residues of active site namely GLY $795 \mathrm{ad}$ GLN585. Other hydrophobic amino acid residues closely placed within the cavity were observed as PHE 798, VAL1200, ALA1198, TYR 592, MET 1038 and ILE1229.

On the other hand, during the visualization of RU3a ${ }_{1}$ the hydrogen bond was observed with $\mathrm{OH}$ group of phenyl ring and hydrophobic residue MET 1038 (Figs. 5, 6). Another hydrogen bond was found similar to $\mathrm{RU}_{3} \mathrm{a}_{3}$ between $\mathrm{OH}$ group of rutinoside and polar residue GLN1194 (Fig. 7). One more hydrogen bonding was observed between one of the $\mathrm{OH}$ group of

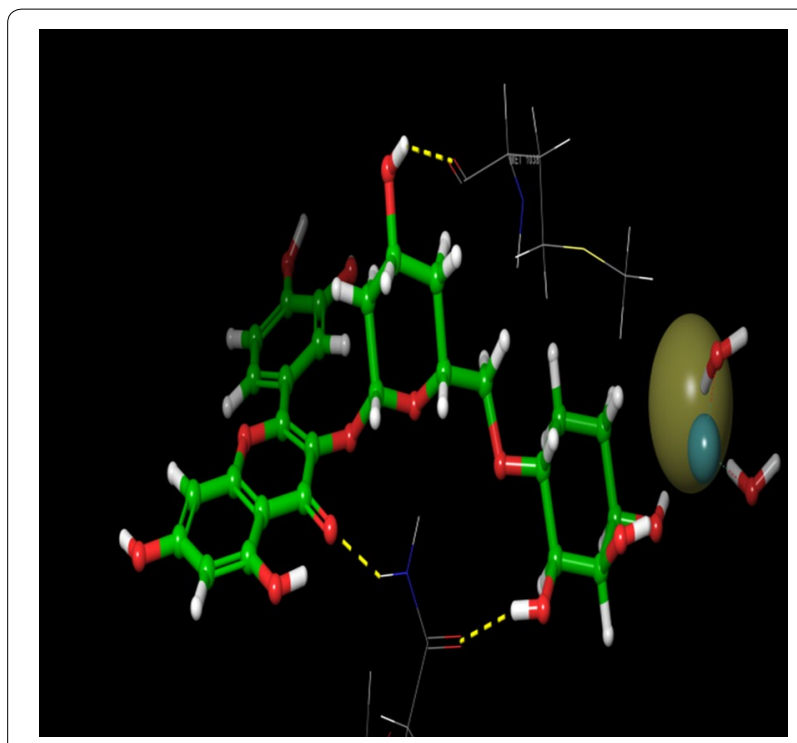

Fig. 8 3D pose of rutin showing hydrogen bonding with GLN 1194 and MET1038

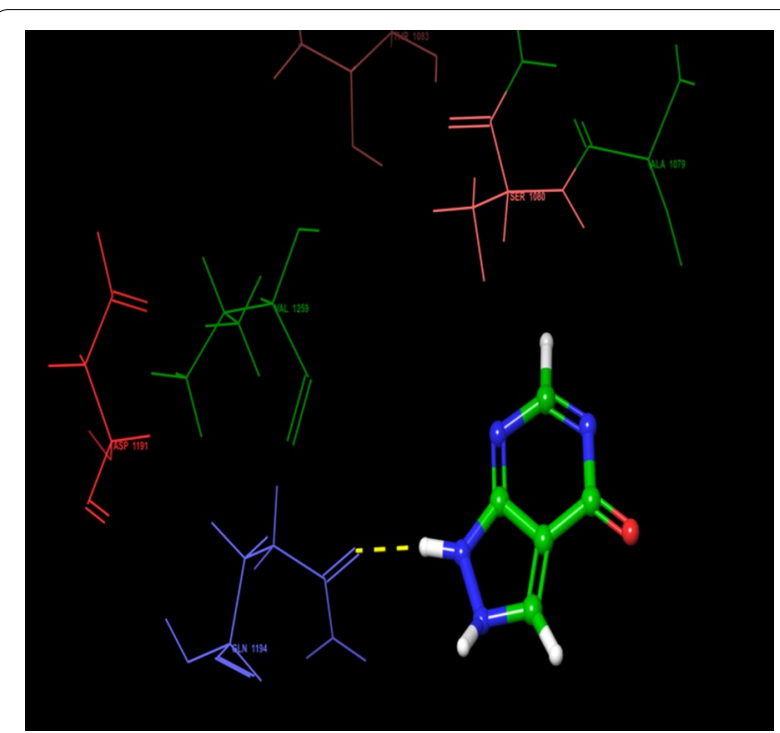

Fig. 9 3D pose of allopurinol showing hydrogen bonding with GLN 1194

dihydroxyphenyl ring and GLY1039. One more interaction was observed with the surrounding residue GLN 767 which forms a hydrogen bond with MOS 1328 (molybdenum metal ion) forming a closed channel to prevent the entry of substrate in the binding site. Other residues surrounding the ligand were observed as ARG 912, HIE 579, GLU 1261, ALA 1189 and ILE1198. When the 3D poses of these two compounds were compared with the native rutin structure, GLN 1194 forms $2 \mathrm{H}$-bonds, one with the $\mathrm{C}=\mathrm{O}$ group of rutin and another with $\mathrm{OH}$ group 
Table 3 In vitro xanthine oxidase inhibitory activity of rutin derivatives

\begin{tabular}{llll}
\hline Compound & $\mathrm{IC}_{\mathbf{5 0}}(\boldsymbol{\mu M}) \pm$ SEM & Compound & $\mathrm{IC}_{\mathbf{5 0}}(\boldsymbol{\mu M}) \pm$ SEM \\
\hline Rutin & $20.867 \pm 0.12$ & RU4b $_{\mathbf{2}}$ & $12.541 \pm 0.45$ \\
RU3a $_{\mathbf{1}}$ & $09.924 \pm 0.01$ & RU7c $_{\mathbf{1}}$ & $19.377 \pm 0.38$ \\
RU3a $_{\mathbf{2}}$ & $07.905 \pm 0.15$ & RU7c $_{\mathbf{2}}$ & $17.428 \pm 0.01$ \\
RU3a $_{\mathbf{3}}$ & $04.870 \pm 0.02$ & RU7c $_{\mathbf{3}}$ & $13.476 \pm 0.25$ \\
RU4b $_{\mathbf{1}}$ & $15.037 \pm 0.01$ & Allopurinol & $10.410 \pm 0.72$ \\
\hline
\end{tabular}

SEM, standard error of the mean

of rutinoside (Fig. 8). The amino acid residues GLU1261 and GLN 1194 were found to be interacted similarly in the study of verbascoside by Wan et al. [40]. Beside this one $\mathrm{H}$-bond was formed between $\mathrm{OH}$ group of chromene ring and MET1038. No pi-pi interaction was in the native structure rutin. In case of Allopurinol, the active site residues surrounding ligand were almost similar and placed near to MOS 1328. The hydrogen bond was observed between purine ring of allopurinol and GLN1194 (Fig. 9).

\section{In-vitro xanthine oxidase inhibitory activity}

In order to monitor the efficacy of different synthesized rutin derivatives, xanthine oxidase inhibitory activity was determined using xanthine oxidase activity assay kit purchased from Sigma-aldrich Co. Allopurinol (positive control) reported to inhibit xanthine oxidase was also screened under identical conditions for comparison. The inhibition ratios revealed the xanthine oxidase inhibitory activity of the synthesized rutin derivatives and the results were summarized in Table 3 . As expected, these rutin derivatives exhibited remarkable activity comparable to the positive control. Based on the in vitro activity; it was observed that hydrazine $\left(R_{3} a_{1}-R_{3} a_{3}\right)$ and anilline analogues (RU4b $\left.b_{1}-R_{4} 4 b_{2}\right)$ were considerably more effective than ester derivatives

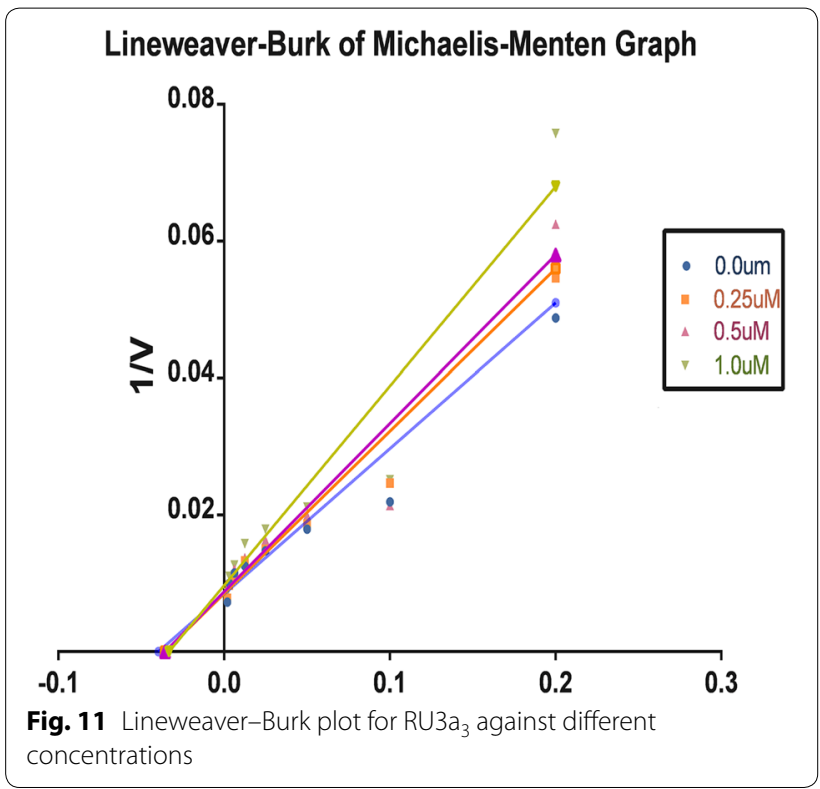

$\left(R_{7} c_{1}-R_{7} c_{3}\right)$. All the compounds of hydrazine

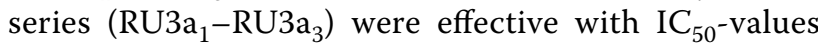
ranging from 04.870 to $09.924 \mu \mathrm{M}$. Rutin hybridized with phenyl hydrazine demonstrated highest activity against xanthine oxidase. While thisemicarbazide and phenylthiosemicarbazide derivatives of rutin showed a slight decrease in activity indicating the role of sulfur group in diminishing the inhibition and $\mathrm{NH}-\mathrm{NH}_{2}$ group in enhancing the activity of targeted enzyme. Surprisingly, substitution of $\mathrm{NH}-\mathrm{NH}_{2}$ with $\mathrm{NH}_{2}$ group leads to decrease of inhibitory activity. Ester derivatives of rutin synthesized after the hydrolysis of rutin exhibited a weaker inhibition than the positive control Allopurinol.

The results of in vitro activity showed $80 \%$ similarity with the results of molecular docking with a few exceptions. In concordance with the screening and output of

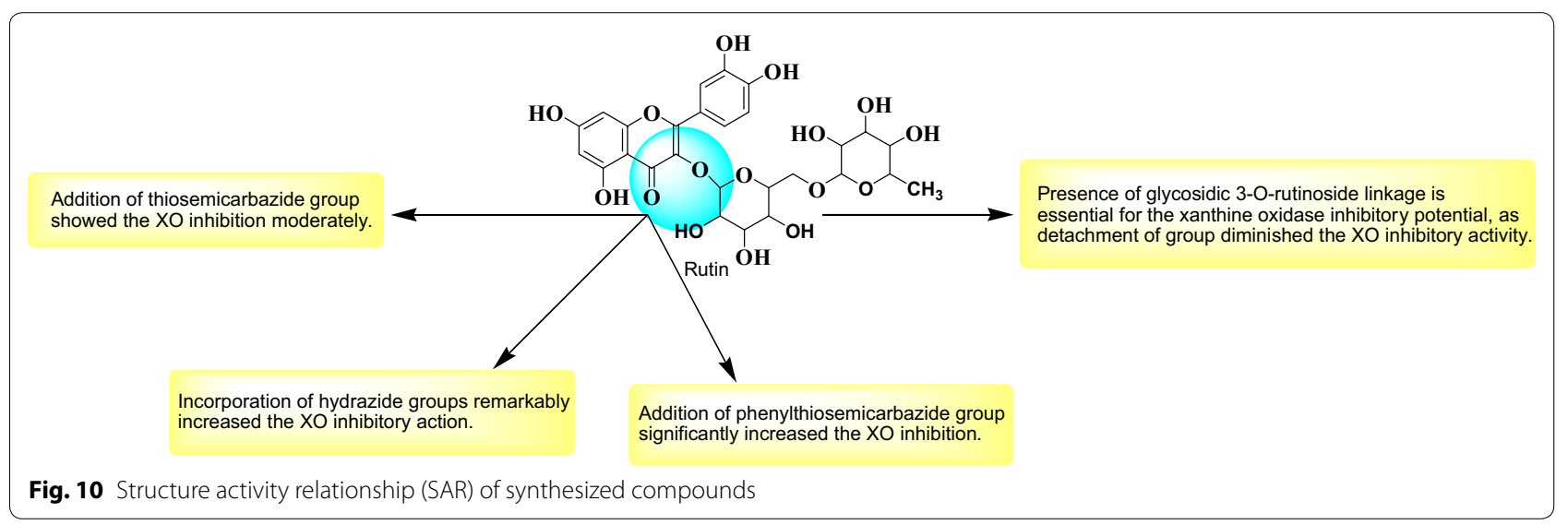




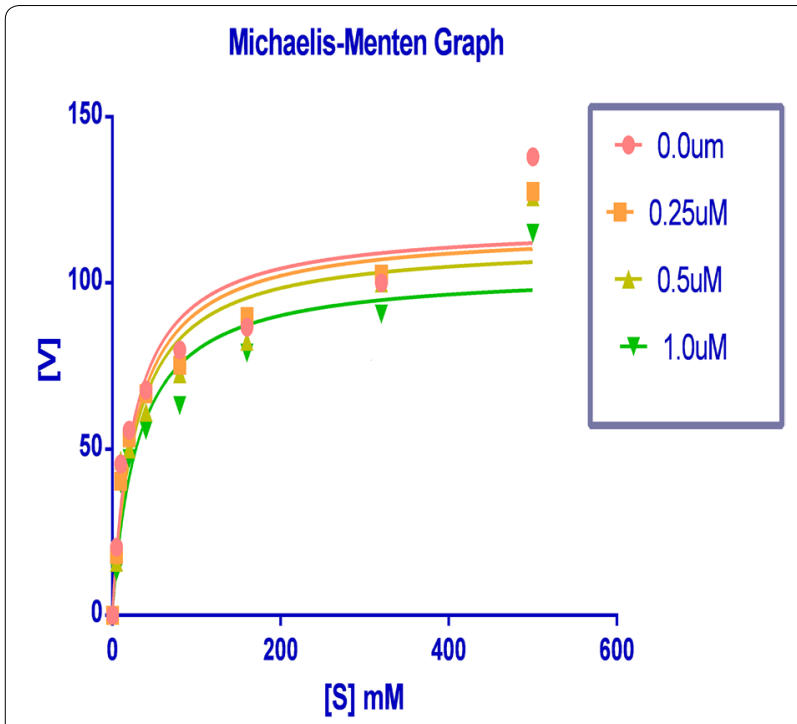

Fig. 12 Michaelis-Menten curve for $\mathrm{RU}_{3} \mathrm{a}_{3}$ at different concentrations

molecular docking $\mathrm{RU}_{3} \mathrm{a}_{3}$ comes out to be most active rutin derivative showing very good interaction with xanthine oxidase at molecular level. Elimination of rutinoside from rutin to synthesize ester derivatives results in a loss of potency with a threefold decrease of inhibitory potential.

\section{Structure activity relationship (SAR)}

Few interesting notions about the relationship of activity and structures of synthesized compounds emerged from the present research (Fig. 10): (A) Rutinoside moiety seems to be important for the activity, as deletion of this leads to loss of activity could be seen from xanthine oxidase inhibitory activity Table 3 . Which shows RU3a $_{3}$ (Having rutinoside group) exhibited highest activity with an IC50 value $04.870 \mu \mathrm{M}$ among all the compounds and $\mathrm{RU}_{7} \mathrm{c}_{1}$ showed lowest activity and fivefold decrease of activity with an $\mathrm{IC}_{50}$ value $19.377 \mu \mathrm{M}$. (B) Hydrazine derivatives were found to be more effective than the aniline derivatives revealing the importance of $\mathrm{NH}-\mathrm{NH}_{2}$ group. But substitution of sulfur group along with hydrazines decreases the activity as in RU3a 3 and RU3a $a_{2}$ and substitution of phenyl group along with sulfur improves the activity $\left(R U 3 a_{1}\right)$. (C) Substitution with ester group leads to a decrease of inhibitory activity.

\section{Enzyme kinetic analysis for XO-inhibitory activity}

To determine the XO-inhibitory mechanisms of newly synthesized derivatives, we carried out kinetic studies
Table $4 K_{m}$ and $V_{\max }$ values of xanthine oxidase at different concentrations of $\mathrm{RU}_{3} \mathrm{a}_{3}$

\begin{tabular}{llll}
\hline S. no. & $\begin{array}{l}\text { Conc. of RU3a } \\
(\mu \mathrm{M})\end{array}$ & $\mathrm{Km}(\mu \mathrm{M})$ & $\mathbf{V}_{\mathbf{m a x}}(\mu \mathrm{mol} / \mathrm{min})$ \\
\hline 1. & 0.0 & 27.21 & 119.6 \\
2. & 0.25 & 30.11 & 114.4 \\
3. & 0.5 & 32.90 & 108.2 \\
4. & 1.0 & 35.08 & 98.7 \\
\hline
\end{tabular}

of most active compound $\mathrm{RU}_{3} \mathrm{a}_{3}$ using Graph pad prism software. Firstly Michaelis-Menten curve was plotted for the enzyme activity at different concentrations of RU3a against different concentration of substrate (xanthine) Fig. 11.

Then double reciprocal plot (Lineweaver-Burk) analysis was done in the presence $(0.25,0.5$, and $1.0 \mu \mathrm{M})$ and absence of $\mathrm{RU}_{3} \mathrm{a}_{3}$ from in vitro data generated during the oxidation of xanthine in presence of xanthine oxidase (Fig. 12). The $\mathrm{x}$ - and $\mathrm{y}$ axis intercepts of the Lineweaver-Burk plot were utilized to calculate $K_{m}$ and $\mathrm{V}_{\text {max }}$ values of $\mathrm{RU}_{3} \mathrm{a}_{3}$ at different concentrations (Table 4).

A concentration-dependent decrease of $V_{\max }$ was predicted in contrast to $\mathrm{K}_{\mathrm{m}}$ value which was found to increasing when concentration of $\mathrm{RU}_{3} \mathrm{a}_{3}$ was increased. The intersection of linear straight lines drawn against each concentration was located at same point, suggesting that $\mathrm{RU}_{3 \mathrm{a}_{3}}$ reacts in competitive manner during the inhibition of xanthine oxidase.

\section{In-vitro evaluation of antioxidant activity by DPPH and $\mathrm{H}_{2} \mathrm{O}_{2}$ method}

The antioxidant potential of newly synthesized compounds was evaluated by DPPH and Hydrogen peroxide radical assay. The comparative analysis of $\mathrm{IC}_{50}$ values for both the assays was done and the results were found to be impressive (Table 5). The results evinced a noteworthy inhibition of DPPH almost all the compounds when compared with the positive control ascorbic acid. In case of DPPH assay compound $\mathrm{RU}_{4} \mathrm{~b}_{1}$ was demonstrated as most potent compound against oxidative stress caused because of free radicals having an $\mathrm{IC}_{50}$ value of $02.647 \pm 0.09 \mu \mathrm{M}$. Along with this compound $\mathrm{RU} 3 \mathrm{a}_{1}$ also showed very good antioxidant potential with an $\mathrm{IC}_{50}$ value of $05.021 \pm 0.10 \mu \mathrm{M}$. When the detailed structure activity relationship was developed between these compounds, it was concluded that both the compounds having hydrazine linkage derived from phenyl hydrazine and phenyl thiosemicarbazide. Similarly, during the analysis of hydrogen peroxide assay all the compounds with hydrazines substitution showed very good antioxidant 
Table 5 Antioxidant activity of synthesized derivatives by DPPH and $\mathrm{H}_{2} \mathrm{O}_{2}$ method

\begin{tabular}{|c|c|c|}
\hline Compound & $\mathrm{IC}_{50}(\mu \mathrm{M}) \pm \mathrm{SEM}$ & $\mathrm{IC}_{50}(\mu \mathrm{M}) \pm \mathrm{SEM}$ \\
\hline$R \cup 3 a_{1}$ & $05.021 \pm 0.10$ & $09.134 \pm 0.35$ \\
\hline $\mathrm{RU} 3 \mathrm{a}_{2}$ & $08.728 \pm 0.02$ & $04.146 \pm 0.01$ \\
\hline $\mathrm{RU}_{3} \mathrm{a}_{3}$ & $11.688 \pm 0.01$ & $06.561 \pm 0.10$ \\
\hline$R \cup 4 b_{1}$ & $02.647 \pm 0.09$ & $09.863 \pm 0.25$ \\
\hline$R \cup 4 b_{2}$ & $08.476 \pm 0.25$ & $04.378 \pm 0.01$ \\
\hline$R \cup 7 c_{1}$ & $06.056 \pm 0.13$ & $14.731 \pm 0.60$ \\
\hline $\mathrm{RUTC}_{2}$ & $14.669 \pm 0.01$ & $12.126 \pm 0.20$ \\
\hline $\mathrm{RU} \mathrm{C}_{3}$ & $07.692 \pm 0.42$ & $17.884 \pm 0.41$ \\
\hline RU001 & $09.483 \pm 0.08$ & $18.623 \pm 0.07$ \\
\hline Ascorbic acid & $22.195 \pm 0.08$ & $22.195 \pm 0.08$ \\
\hline
\end{tabular}

SEM, standard error of the mean

potential having $\mathrm{IC}_{50}$ in range of $04.146 \pm 0.01$ to $09.134 \pm 0.35$ (Fig. 7). Compound $\mathrm{RU}_{3} \mathrm{a}_{2}$ having phenyl thiosemicarbazide substitution showed potential antioxidant activity among all the derivatives. Along with this phenyl hydrazine substituted rutin derivative ( $\left.\mathrm{RU}_{3} \mathrm{a}_{3}\right)$ also showed very good scavenging activity with an $\mathrm{IC}_{50}$ value of $06.561 \pm 0.10$. When the detailed structure activity relationship was developed between these compounds, it was concluded that both the compounds having hydrazine linkage derived from phenyl hydrazine and phenyl thiosemicarbazide.

\section{Conclusion}

Starting from the structures of rutin as anti-XO hit previously identified, different series of novel analogues were designed and synthesized to explore the structure-activity relationships associated with these xanthine oxidase inhibitors along with their antioxidant potential. Different structural elements were identified as essential for antioxidant and anti-XO properties, such as the presence of rutinoside (RU3a $\mathrm{a}_{1}, \mathrm{RU} 3 \mathrm{a}_{2}$ and RU3a $\mathrm{a}_{3}$ ) comes out as important skeleton for the inhibitory potential, presence of hydrazone linker along with phenyl group, while the associated xanthine oxidase inhibitory effect was found to follow a different trend for the two series hydrazine $\left(\mathrm{RU}_{3 \mathrm{a}_{1-3}}\right)$ and ester derivatives $\left(\right.$ RU7c $\left._{1-3}\right)$. The newly synthesized derivatives with antioxidant and ani-XO $\mathrm{IC}_{50}$ values in the low micromolar range and good selectivity indexes were identified. Contemporary synthetic efforts are focused towards the insertion of the hydrazones and ester linkage by replacing the side linkage rutinoside of rutin with more stable groups while maintaining the overall length of new derivatives. Molecular docking provide an improved trail to design the new molecules with an avantgarde stability and potency.

\section{Additional file}

Additional file 1. HNMR spectra of compound $R U 3 a_{3}$

\section{Acknowledgements}

The authors are highly thankful to the Head, Department of Pharmaceutical Sciences, M. D. University, Rohtak for providing essential facilities to accomplish this research study. The authors are also thankful to Dr. Vinod Devaraji Application Scientist Schrödinger LLC for his support to carry out the computational work.

\section{Authors' contributions}

Authors NM and AK have designed, synthesized and carried out the xanthine oxidase inhibitory and antioxidant activity and the author PD, have carried out the docking simulations with in silico ADMET studies. All authors read and approved the final manuscript.

\section{Funding}

No funding received for this research work from outside sources.

Availability of data and materials

Not applicable.

\section{Competing interests}

The authors declare that they have no competing interests.

\section{Author details}

${ }^{1}$ Faculty, Department of Pharmaceutical Sciences, M.D. University, Rohtak 124001, India. ${ }^{2}$ Laboratory for Preservation Technology and Enzyme Inhibition Studies, Department of Pharmaceutical Sciences, M.D. University, Rohtak, Haryana, India.

Received: 21 January 2019 Accepted: 2 May 2019

Published online: 23 May 2019

References

1. Berry CE, Hare JM (2004) Xanthine oxidoreductase and cardiovascular disease: molecular mechanisms and pathophysiological implications. J Physiol 555(3):589-606

2. Moriwaki Y, Yamamoto T, Higashino K (1997) Distribution and pathophysiologic role of molybdenum-containing enzymes. Histol Histopathol 12(2):513-524

3. Klinenberg JR, Goldfinger SE, Seegmiller JE (1965) The effectiveness of the xanthine oxidase inhibitor allopurinol in the treatment of gout. Ann Intern Med 62(4):639-647

4. Yu KH (2007) Febuxostat: a novel non-purine selective inhibitor of xanthine oxidase for the treatment of hyperuricemia in gout. Recent Pat Inflamm Allergy Drug Discov 1(1):69-75

5. Battelli MG, Bolognesi A, Polito L (2014) Pathophysiology of circulating xanthine oxidoreductase: new emerging roles for a multi-tasking enzyme. Biochim Biophys Acta Mol Basis Dis 1842(9):1502-1517

6. Brass CA, Narciso J, Gollan JL (1991) Enhanced activity of the free radical producing enzyme xanthine oxidase in hypoxic rat liver. Regulation and pathophysiologic significance. J Clin Invest 87(2):424-431

7. Chambers DE, Parks DA, Patterson G, Roy R, McCord JM, Yoshida S, Parmley LF, Downey JM (1985) Xanthine oxidase as a source of free radical damage in myocardial ischemia. J Mol Cell Cardiol 17(2):145-152

8. Desco MC, Asensi M, Márquez R, Martínez-Valls J, Vento M, Pallardó FV, Sastre J, Viña J (2002) Xanthine oxidase is involved in free radical production in type 1 diabetes: protection by allopurinol. Diabetes 51(4):1118-1124

9. Kuppusamy P, Zweier JL (1989) Characterization of free radical generation by xanthine oxidase. Evidence for hydroxyl radical generation. J Biol Chem 264(17):9880-9884

10. Dawson J, Walters M (2006) Uric acid and xanthine oxidase: future therapeutic targets in the prevention of cardiovascular disease? $\mathrm{Br} J$ Clin Pharmacol 62(6):633-644 
11. Khosla UM, Zharikov S, Finch JL, Nakagawa T, Roncal C, Mu W, Krotova K, Block ER, Prabhakar S, Johnson RJ (2005) Hyperuricemia induces endothelial dysfunction. Kidney Int 67(5):1739-1742

12. Kaynar H, Meral M, Turhan H, Keles M, Celik G, Akcay F (2005) Glutathione peroxidase, glutathione-S-transferase, catalase, xanthine oxidase, $\mathrm{Cu}-\mathrm{Zn}$ superoxide dismutase activities, total glutathione, nitric oxide, and malondialdehyde levels in erythrocytes of patients with small cell and non-small cell lung cancer. Cancer Lett 227(2):133-139

13. Griguer CE, Oliva CR, Kelley EE, Giles GI, Lancaster JR, Gillespie GY (2006) Xanthine oxidase-dependent regulation of hypoxia-inducible factor in cancer cells. Cancer Res 66(4):2257-2263

14. Kanellis J, Kang DH (2005) Uric acid as a mediator of endothelial dysfunction, inflammation, and vascular disease. Seminars in nephrology, vol 25. New York, WB Saunders, pp 39-42

15. Miesel R, Zuber M (1993) Elevated levels of xanthine oxidase in serum of patients with inflammatory and autoimmune rheumatic diseases. Inflammation 17(5):551-561

16. Wijermars LG, Bakker JA, de Vries DK, van Noorden CJ, Bierau J, Kostidis S, Mayboroda OA, Tsikas D, Schaapherder AF, Lindeman JH (2016) The hypoxanthine-xanthine oxidase axis is not involved in the initial phase of clinical transplantation-related ischemia-reperfusion injury. Am J Physiol Renal Physiol 312(3):F457-F464

17. Poles MZ, Bódi N, Bagyánszki M, Fekete É, Mészáros AT, Varga G, Szűcs S, Nászai A, Kiss L, Kozlov AV, Boros M (2018) Reduction of nitrosative stress by methane: neuroprotection through xanthine oxidoreductase inhibition in a rat model of mesenteric ischemia-reperfusion. Free Radic Biol Med 120:160-169

18. Osada Y, Tsuchimoto M, Fukushima H, Takahashi K, Kondo S, Hasegawa $M$, Komoriya K (1993) Hypouricemic effect of the novel xanthine oxidase inhibitor, TEl-6720, in rodents. Eur J of Pharmacol 241(2-3):183-188

19. Krakoff IH, Meyer RL (1965) Prevention of hyperuricemia in leukemia and lymphoma: use of allopurinol, a xanthine oxidase inhibitor. JAMA 193(1):1-6

20. Pacher PA, Nivorozhkin A, Szabó C (2006) Therapeutic effects of xanthine oxidase inhibitors: renaissance half a century after the discovery of allopurinol. Pharmacol Rev 58(1):87-114

21. Inkster ME, Cotter MA, Cameron NE (2007) Treatment with the xanthine oxidase inhibitor, allopurinol, improves nerve and vascular function in diabetic rats. Eur J Pharmacol 561(1-3):63-71

22. Sagor M, Taher A, Tabassum N, Potol M, Alam M (2015) Xanthine oxidase inhibitor, allopurinol, prevented oxidative stress, fibrosis, and myocardial damage in isoproterenol induced aged rats. Oxid Med Cell Longev. https ://doi.org/10.1155/2015/478039

23. Min HK, Lee B, Kwok SK, Ju JH, Kim WU, Park YM, Park SH (2015) Allopurinol hypersensitivity syndrome in patients with hematological malignancies: characteristics and clinical outcomes. Korean J Intern Med 30(4):521

24. Quach C, Galen BT (2018) HLA-B* 5801 testing to prevent allopurinol hypersensitivity syndrome: a teachable moment. JAMA Int Med 178(9):1260-1261

25. Takano Y, Hase-Aoki K, Horiuchi H, Zhao L, Kasahara Y, Kondo S, Becker MA (2005) Selectivity of febuxostat, a novel non-purine inhibitor of xanthine oxidase/xanthine dehydrogenase. Life Sci 76(16):1835-1847

26. Mayer MD, Khosravan R, Vernillet L, Wu JT, Joseph-Ridge N, Mulford DJ (2005) Pharmacokinetics and pharmacodynamics of febuxostat, a new non-purine selective inhibitor of xanthine oxidase in subjects with renal impairment. Am J Ther 12(1):22-34

27. Nepali K, Singh G, Turan A, Agarwal A, Sapra S, Kumar R, Banerjee UC, Verma PK, Satti NK, Gupta MK, Suri OP (2011) A rational approach for the design and synthesis of 1-acetyl-3, 5-diaryl-4, 5-dihydro $(1 \mathrm{H})$ pyrazoles as a new class of potential non-purine xanthine oxidase inhibitors. Bioorg Med Chem 19(6):1950-1958

28. Becker MA, Kisicki J, Khosravan R, Wu J, Mulford D, Hunt B, MacDonald P, Joseph-Ridge N (2004) Febuxostat (TMX-67), a novel, non-purine, selective inhibitor of xanthine oxidase, is safe and decreases serum urate in healthy volunteers. Nucleosides Nucleotides Nucleic Acids 23(8-9):1111-1116
29. Khosravan R, Grabowski BA, Wu JT, Joseph-Ridge N, Vernillet L (2006) Pharmacokinetics, pharmacodynamics and safety of febuxostat, a nonpurine selective inhibitor of xanthine oxidase, in a dose escalation study in healthy subjects. Clin Pharmacokinet 45(8):821-841

30. Malik N, Dhiman P, Khatkar A (2017) In-silico design and ADMET studies of natural compounds as inhibitors of xanthine oxidase $(X O)$ enzyme. Curr Drug Metab 18(6):577-593

31. Muhammad A, Arthur DE, Babangida S, Erukainure OL, Malami I, Sani H, Abdulhamid AW, Ajiboye IO, Saka AA, Hamza NM, Asema S (2018) Modulatory role of rutin on 2, 5-hexanedione-induced chromosomal and DNA damage in rats: validation of computational predictions. Drug Chem Toxicol 10:1-4

32. Roleira FM, Varela CL, Costa SC, Tavares-da-Silva EJ (2018) Phenolic derivatives from medicinal herbs and plant extracts: anticancer effects and synthetic approaches to modulate biological activity. Nat Prod Chem 57:115-156

33. Baldisserotto A, Vertuani S, Bino A, De Lucia D, Lampronti I, Milani R, Gambari R, Manfredini S (2015) Design, synthesis and biological activity of a novel Rutin analogue with improved lipid soluble properties. Bioorg Med Chem 23(1):264-271

34. Gullon B, Lú-Chau TA, Moreira MT, Lema JM, Eibes G (2017) Rutin: a review on extraction, identification and purification methods, biological activities and approaches to enhance its bioavailability. Trends Food Sci Technol 67:220-235

35. Friesner RA, Banks JL, Murphy RB, Halgren TA, Klicic JJ, Mainz DT, Repasky MP, Knoll EH, Shelley M, Perry JK, Shaw DE (2004) Glide: a new approach for rapid, accurate docking and scoring. 1. Method and assessment of docking accuracy. J Med Chem 47(7):1739-1749

36. Dhiman P, Malik N, Verma PK, Khatkar A (2015) Synthesis and biological evaluation of thiazolo and imidazo N-(4-nitrophenyl)-7-methyl5-aryl-pyrimidine-6 carboxamide derivatives. Res Chem Intermed 41(11):8699-8711

37. Patel A, Patel A, Patel A, Patel NM (2010) Determination of polyphenols and free radical scavenging activity of Tephrosia purpurea linn leaves (Leguminosae). Pharmacogn Res 2:152-154

38. Li P, Tian Y, Zhai H, Deng F, Xie M, Zhang X (2013) Study on the activity of non-purine xanthine oxidase inhibitor by 3D-QSAR modeling and molecular docking. J Mol Struct 5(1051):56-65

39. Shen L, Ji HF (2009) Insights into the inhibition of xanthine oxidase by curcumin. Bioorg Med Chem Lett 19(21):5990-5993

40. Wan Y, Zou B, Zeng H, Zhang L, Chen M, Fu G (2016) Inhibitory effect of verbascoside on xanthine oxidase activity. Int J Biol Macromol 1(93):609-614

\section{Publisher's Note}

Springer Nature remains neutral with regard to jurisdictional claims in published maps and institutional affiliations.

Ready to submit your research? Choose BMC and benefit from

- fast, convenient online submission

- thorough peer review by experienced researchers in your field

- rapid publication on acceptance

- support for research data, including large and complex data types

- gold Open Access which fosters wider collaboration and increased citations

- maximum visibility for your research: over 100M website views per year

At BMC, research is always in progress.

Learn more biomedcentral.com/submissions 